\title{
Interactions of the solutions of a coupled system of Benjamin-Bona-Mahony type in weighted spaces
}

DOI: $10.46932 / \mathrm{sfjdv} 2 \mathrm{n} 1-058$

Received in: November 1st, 2020

Accepted in: December 30th, 2020

\author{
Hernan Oscar Cortez Gutiérrez \\ Universidad Nacional del Callao (UNAC), Callao, Perú \\ E-mail: hocortezg@unac.edu.pe \\ Milton Milciades Cortez Gutiérrez \\ Department of Mathematics \\ National University of Trujillo-(UNT), Trujillo, Peru \\ E-mail: mcortezgutierrez@yahoo.es \\ Girady Iara Cortez Fuentes Rivera \\ Universidad Nacional del Callao (UNAC), Callao, Perú \\ E-mail: gicortezf@unac.edu.pe \\ Liv Jois Cortez Fuentes Rivera \\ Universidad Privada San Juan Bautista (UPSJB), Lima, Perú \\ E-mail: livjoisc@gmail.com \\ Deolinda Ellyda Fuentes Rivera Vallejo \\ Universidad Inca Garcilaso de la Vega, Lima, Perú \\ E-mail: deitofuentesriva@ outlook.es
}

\begin{abstract}
Interactions of the solutions are a part of collisions of wave systems. We prove the existence of the solutions of a coupled system of Benjamin- Bona-Mahony (BBM) type in a weighted Sobolev space with initial data. we are concerned with a dispersive system for an appropriate class of nonlinearities. The study for a coupled system of BBM type without weighted was initiated by Bisognin,V. [3] Further results can be found in Bona,J.-Ponce,G.-Saut,J.C. [4] .
\end{abstract}

Keywords: global existence, coupled system, weighted Sobolev.

\section{INTRODUCTION}

Recently Chen and Wang [1] studied the stability of the solitary wave solutions to a coupled BBM system

$$
\begin{aligned}
& u_{t}-u_{x x t}+u_{x}++P(u, v)_{x}=0 \\
& v_{t}-v_{x x t}+v_{x}+Q(u, v)_{x}=0 \\
& \text { In }-\infty<x<\infty, t>0
\end{aligned}
$$

We introduce the coupled system of Benjamin- Bona-Mahony type in a weighted Sobolev: 


$$
\begin{gathered}
u_{t}-u_{x x t}-a v_{x x t}+a_{1} u_{x}+a_{2} v^{p} v_{x}+u^{p} u_{x}+a_{3}\left(u^{p} v\right)_{x}=0 \\
v_{t}-v_{x x t}-a u_{x x t}+a_{1} v_{x}+a_{2} u^{p} u_{x}+v^{p} v_{x}+a_{3}\left(u v^{p}\right)_{x}=0 \\
\text { In }-\infty<x<\infty, t>0
\end{gathered}
$$

Here $a, a_{1}, a_{2}$ and $a_{3}$ are constants. Assume now that $a_{1}>0,0<a<1$ and $p \in \mathbb{N}$ with $p>1$; the unknowns are $u, v$ defined in $\mathbb{R}^{2+}=(-\infty, \infty) \times[0, \infty)$. We consider the initial conditions

$$
u(x, 0)=u_{0}(x) \text { and } v(x, 0)=v_{0}(x)
$$

for the coupled system (1.1). Let us first introduce some notation. Let $X_{r}=H_{r}^{2}(\mathbb{R}) \times H_{r}^{2}(\mathbb{R})$. Where $H_{r}^{2}(\mathbb{R})$ is a Hilbert space with the inner product $(u, v)_{r, 2}=\left(M_{r} \Lambda^{2} u, M_{r} \Lambda^{2} v\right)_{2}$. Here $(., .)_{2}$ is the inner product in $L^{2}(\mathbb{R})$. For $r \in \mathbb{R}^{+}$, let $\Lambda^{2}$ be the Pseudo-differential operator defined by $\Lambda^{2}=\left(I-\partial_{x}^{2}\right)$. Furthermore, we set $M_{r} f(x)=\left(1+|\mathrm{x}|^{2}\right)^{r / 2} f(x)$. In order to apply semigroup theory, we must first transform the coupled system (1.1) to a first-order system. For this purpose, We set

$$
\begin{gathered}
A=\left(\begin{array}{cc}
\Lambda^{2} & -a \partial_{x}^{2} \\
-a \partial_{x}^{2} & \Lambda^{2}
\end{array}\right), B=\left(\begin{array}{cc}
a_{1} & 0 \\
0 & a_{1}
\end{array}\right), w=\left(\begin{array}{l}
u \\
v
\end{array}\right) \\
(F w)_{x}=\left(\begin{array}{l}
\left(a_{2} \frac{\mathrm{v}^{p+1}}{p+1}+\frac{\mathrm{u}^{p+1}}{p+1}+a_{3}\left(u^{p} v\right)_{x}\right)_{x} \\
\left(a_{2} \frac{\mathrm{u}^{p+1}}{p+1}+\frac{\mathrm{v}^{p+1}}{p+1}+a_{3}\left(v^{p} u\right)_{x}\right)_{x}
\end{array}\right), \text { and we rewrite (1.1) and (1.2) as } \\
\left\{\begin{array}{l}
A w_{t}+B w_{x}+(F w)_{x}=0, x \in \mathbb{R}, t>0 \\
w(x, 0)=w_{0}(x) \quad, \quad x \in \mathbb{R}
\end{array}\right. \\
\text { Where } w_{0}=\left(\begin{array}{l}
u_{0} \\
v_{0}
\end{array}\right) .
\end{gathered}
$$

\subsection{PRELIMINARIES}

Let $X$ a Hilbert space and $m \in \mathbb{N}, T>0$ we denote by $C^{m}([0, T] ; X)$ the space of vector. Valued functions $u:[0, T] \rightarrow X$ m-times continuously differentiable in $[0, T]$ with the usual norm:

$$
\mu(x)=\left(1+|x|^{2}\right)^{1 / 2}
$$

For every $s \in \mathbb{R}$, the Sobolev spaces $H^{S}(\mathbb{R})$ are introduced as usual with the norm

$\|u\|_{s}=\left|\mu^{s} \hat{u}\right|_{2}$ where the $|$.$| denotes the standard norm of L^{2}(\mathbb{R})$ and $\hat{u}$ is the Fourier transform of $u$. We also denote by 


$$
\Lambda^{s}=\left(I-\partial_{x}^{2}\right)^{s / 2}
$$

The Bessel potential of order $s$.

If $r \in \mathbb{R}$ we denote by $M_{r}$ the multiplication operator with the function $\mu^{r}(x)$. While if $r, s \in \mathbb{R}$ we denote by $H_{r}^{s}(\mathbb{R})=H^{s}\left(\mathbb{R}, \mu^{r}(x) d x\right)$ the completion of Schwarz space $\mathcal{S}(\mathbb{R})$ in the norm

$$
|u|_{r, s}=\left|M_{r} \Lambda^{s} u\right|_{2}
$$

$H_{r}^{S}(\mathbb{R})$ is a Hilbert space with the inner product

$$
(u, v)_{r, s}=\left(M_{r} \Lambda^{s} u, M_{r} \Lambda^{s} v\right)_{2} \text { and its dual is defined by }\left(H_{r}^{s}(\mathbb{R})\right)^{\prime}=H_{-r}^{-s}(\mathbb{R}) \text {. When } r=0 \text {, the space }
$$

$H_{0}^{S}$ coincide with the usual norm Sobolev space. The case $r=s=0$ we put $H_{0}^{0}(\mathbb{R})=L^{2}(\mathbb{R})$.

We introduce the Hilbert space $\mathcal{S}_{r}^{S}(\mathbb{R})$ defined by

$$
\mathcal{S}_{r}^{s}(\mathbb{R})=H_{r}^{0}(\mathbb{R}) \cap H_{0}^{s}(\mathbb{R})
$$

For every $r, s \in \mathbb{R}$ with the inner product

$$
(((u, v)))_{r, s}=(u, v)_{r, 0}+(u, v)_{0, s}
$$

We give some properties

i)For evey $r_{1}, s_{1}, r_{2}, s_{2} \in \mathbb{R}$ and $\theta \in(0,1)$

$$
\left[H_{r_{1}}^{s_{1}}, H_{r_{2}}^{s_{2}}\right]_{\theta}=H_{(1-\theta) r_{1}+\theta r_{2}}^{(1-\theta) s_{1}+\theta s_{2}}
$$

Where $[X, Y]_{\theta}$ is the interpolation space of $\mathrm{X}$ and $\mathrm{Y}$.

ii) For every $r, s \in \mathbb{R}$ there exists $c_{1}, c_{2}$ positive constants such that

$$
c_{1}|u|_{r, s} \leq\left|\mathcal{F}^{-1}\left(M_{s} \mathcal{F}\left(M_{r} u\right)\right)\right|_{2} \leq c_{2}|u|_{r, s}
$$

For every $s \in H_{r}^{s}(\mathbb{R})$

iii) For every $r, s \in \mathbb{R}, \quad \mathcal{F}\left(H_{r}^{s}(\mathbb{R})\right)=H_{s}^{r}(\mathbb{R})$

iv) If $r_{1} \geq r_{2}, s_{1} \geq s_{2}$ then 


$$
H_{r_{1}}^{s_{1}} \subset H_{r_{2}}^{s_{2}}, \quad \delta_{r_{1}}^{s_{1}} \subset \mathcal{S}_{r_{2}}^{s_{2}} \text { with continuous injection. }
$$

v) For every $r, s \in \mathbb{R}$ and $\theta \in(0,1)$

$$
\mathcal{S}_{r}^{S}(\mathbb{R}) \subset\left[H_{r}^{0}(\mathbb{R}), H_{0}^{s}(\mathbb{R})\right]_{\theta}=H_{(1-\theta) r}^{\theta s}
$$

vi) If $r_{1}, s_{1}, r_{2}, s_{2}, r, s \in \mathbb{R}$ and $u \in H_{r_{1}}^{s_{1}}(\mathbb{R}), v \in H_{r_{2}}^{s_{2}}(\mathbb{R})$ then $u v \in H_{r}^{s}(\mathbb{R})$ whenever

$$
s_{1}, s_{2} \geq s, \quad s_{1}+s_{2}-s \geq 1 / 2
$$

Moreover there exists a constant $c>0$ such that:

$$
|u v|_{r, s} \leq c|u|_{r_{1}, s_{1}}|u|_{r_{2}, s_{2}}
$$

For some other details one find in (cf.[4],[14].

\section{RESULTS}

\subsection{Global existence and mild solution}

Theorem 1. Let $r \geq 0$ if $u_{0} \in H^{4}(\mathbb{R}) \cap H_{r}^{2}(\mathbb{R})$

then the initial value problem

$$
\left\{\begin{array}{c}
\partial_{t} u+\partial_{x} u+u \partial_{x} u-\partial_{x}^{2} \partial_{t} u=0, x \in \mathbb{R}, t>0 \\
u(x, 0)=u_{0}(x), \quad x \in \mathbb{R}
\end{array}\right.
$$

Has a unique solution

$$
u \in C^{0}\left([0, \infty) ; H^{4}(\mathbb{R}) \cap H_{r}^{2}(\mathbb{R})\right) \cap C^{1}\left([0, \infty) ; H_{r}^{2}(\mathbb{R})\right)
$$

Before proceeding to the proof, we establish some preliminary results. We know that (2) is equivalent to the following initial-value problem:

$$
\left\{\begin{array}{c}
\partial_{t} u=B u+B f u, x \in \mathbb{R}, t>0 \\
u(x, 0)=u_{0}(x), \quad x \in \mathbb{R}
\end{array}\right.
$$

Where $B=-\Lambda^{-2} \partial_{x}, f(s)=s^{2} / 2$ 
Lemma1. $B: H_{r}^{2}(\mathbb{R}) \rightarrow H_{r}^{2}(\mathbb{R})$ is a linear bounded operator.

Proof. In fact, for $u \in H_{r}^{2}(\mathbb{R})$ we have

$$
\begin{gathered}
|B u|_{r, 2}^{2}=\left|M_{r} \Lambda^{2} \Lambda^{-2} \partial_{y} u\right|_{2}^{2}=\left|M_{r} \partial_{y} u\right|_{2}^{2}= \\
\int_{\mathbb{R}}\left(1+|y|^{2}\right)^{r} \partial_{y} u \partial_{y} u d y=- \\
-\int_{\mathbb{R}} u \partial_{y}\left(\left(1+|y|^{2}\right)^{r} \partial_{y} u\right) d y=- \\
-\int_{\mathbb{R}} 2 r\left(1+|y|^{2}\right)^{r-1} y u \partial_{y} u d y- \\
-\int_{\mathbb{R}}\left(1+|y|^{2}\right)^{r} u_{y y} u d y
\end{gathered}
$$

Since that $y^{2} \leq\left(1+|y|^{2}\right)^{2}$

It follows from the Cauchy-Schwarz's inequality

$$
\begin{gathered}
|B u|_{r, 2}^{2} \leq \\
2 r\left|M_{r} \partial_{y} u\right|_{2} \cdot\left|M_{r} u\right|_{2}+\left|M_{r} u\right|_{2}\left|M_{r} \partial_{y}^{2} u\right|_{2}
\end{gathered}
$$

On the other hand, an elementary calculation based on the identity

$$
\begin{array}{r}
\left|\Lambda^{2} u\right|^{2}=\left|\left(I-\partial_{y}^{2}\right) u\right|^{2}=|u|^{2}+ \\
+\left|\partial_{y}^{2} u\right|^{2}-2 u \partial_{y}^{2} u
\end{array}
$$

Shows that

$$
\left|\partial_{y}^{2} u\right|_{r, 0}^{2} \leq c|u|_{r, 0}^{2}+|u|_{r, 2}^{2}
$$

And so, by applying the embedding $H_{r}^{2} \subset H_{r}^{0}$

It follows that 


$$
|B u|_{r, 2}^{2}=\left|\partial_{y}^{2} u\right|_{r, 0}^{2} \leq c|u|_{r, 2}^{2}+|u|_{r, 2}^{2}
$$

Hence

$$
|B u|_{r, 2} \leq c|u|_{r, 2}
$$

1 Proposition1. (local existence) Given $u_{0} \in H_{r}^{2}(\mathbb{R})$ there exists $T \in(0, \infty)$ and an unique mild solution $u$ of (1) in $\mathbb{R} \times(0, T)$ with initial data $u_{0}$. Here a mild solution is an element of $C\left([0, T] ; H_{r}^{2}(\mathbb{R})\right)$ satisfying

$$
u(t)=e^{B t} u_{0}+\int_{0}^{t} e^{B(t-\sigma)} B\left(\frac{u(\sigma)^{2}}{2}\right) d \sigma
$$

We use Lemmal for concluding that $\mathrm{B}$ is the infinitesimal generator of a uniformly continuous semigroup of operators on $H_{r}^{2}(\mathbb{R})$. The conclusion follows using standard arguments given in Pazy[7].

\section{Proof of Theorem 1}

Note that $B f$ is differentiable, $u(x, t)$ is a classical solution of (1) on any $t$-interval where it exists. Furthermore

$$
\partial_{t} u=B u+B f u \in C\left([0, T] ; H_{r}^{2}(\mathbb{R})\right)
$$

For every $s \geq 0$ the existence of a global $H^{s}$ - valued solution for (1) has already been proved (cf.[1],[2]). In addition such solution belongs to $C^{0}\left([0, \infty) ; H^{s}(\mathbb{R})\right)$.

We claim that for every $T>0$, there exists some constant $M_{T}>0$ such that

$$
\sup _{0 \leq t \leq T}|u(., t)|_{r, 2} \leq M_{T}
$$

In fact, for every $u_{0} \in H_{r}^{2}(\mathbb{R})$ by the density of $\mathcal{S}_{2 r}^{4}(\mathbb{R})$ in $H_{r}^{2}(\mathbb{R})$ (cf.[14])there exists

$\left(u_{0 n}\right) \in \mathcal{S}_{2 r}^{4}(\mathbb{R})$ such that

$u_{0 n} \rightarrow u_{0}$ strong in $H_{r}^{2}(\mathbb{R})$

Now consider $u_{n}=u_{n}(x, t)$ be the solution of initial value problem.

$$
\left\{\begin{array}{c}
\partial_{t} u_{n}+\partial_{x} u_{n}+u \partial_{x} u_{n}-\partial_{x}^{2} \partial_{t} u_{n}=0, x \in \mathbb{R}, t>0 \\
u_{n}(x, 0)=u_{0 n}(x), \quad x \in \mathbb{R}
\end{array}\right.
$$

Then the solution $u_{n}$ exists globally in $C\left([0, \infty) ; \mathcal{S}_{2 r}^{4}(\mathbb{R})\right)$, (cf.[13]). In particular given any $T>0$ there exists a constant $M_{T}$ (independent of $n$ ) such that 


$$
\left\|\mid u_{n}\right\|_{2 r, 4} \leq M_{T}
$$

And $\left(u_{n}\right)$ converges to the solution $u=u(x, t)$ of the problem (2). Since we have the embedding injection $\mathcal{S}_{2 r}^{4}(\mathbb{R}) \subset H_{r}^{2}(\mathbb{R})$ it follows that

$$
\left|u_{n}(., t)\right|_{r, 2} \leq C||\left|u_{n}\right|||_{2 r, 4} \leq M_{T}
$$

Finally, we claim that

$$
|u(., t)|_{r, 2} \leq \lim _{n \rightarrow \infty} \inf \left|u_{n}(., t)\right|_{r, 2} \leq M_{T}
$$

This completes the proof.

\subsection{LEMMAS FOR THE BBM SYSTEM}

Lemma 2.1 If $A$ is as above, and let $\eta>0, \delta>0, r \geq 0$ such that $0<a<\min \left\{1, \frac{\delta}{\eta \delta+C_{r}}, \frac{\eta}{\eta \delta+C_{r}}\right\}$ for some $C_{r}>0$, then $A$ is invertible and bounded below.

Lemma 2.2 Let $g=\left(g_{1}, g_{2}\right) \in X_{r}$, and consider the assumptions of Lemma 2.1. Then, the following holds.

i) $\quad K * \frac{\partial g}{\partial x} \in X_{r}$, and there exists $C>0$ such that $\left|K * \frac{\partial g}{\partial x}\right|_{X_{r}} \leq C|g|_{X_{r}}$

ii) $\quad A^{-1} g=K * g$, where $K=\left(K_{m n}\right)_{1 \leq m, n \leq 2}$ and each of the $K_{m n}$ 's satisfies

$$
\begin{gathered}
K_{m n}=\frac{1}{\sqrt{2 \pi}} \int_{\mathbb{R}} e^{i x y} a_{m n}(y) d y, i=\sqrt{-1} \text { and we set } \\
\widehat{A^{-1} g}(y)=\left(a_{m n}(y)\right) \hat{g}(y), \hat{g}(y)=\left(\begin{array}{l}
\hat{g}_{1}(y) \\
\hat{g}_{2}(y)
\end{array}\right)
\end{gathered}
$$

Theorem 1.1 Let $w_{0}=\left(u_{0}, v_{0}\right) \in X_{r}$ be as above and suppose the assumptions from Lemma 2.1 hold. Then there exists a unique solution of the coupled system (1.1) and (1.2) satisfying $w \in$ $C^{0}\left(\left[0, T_{0}\right] ; H_{r}^{2}(\mathbb{R})\right) \times C^{0}\left(\left[0, T_{0}\right] ; H_{r}^{2}(\mathbb{R})\right)$, for some $T_{0}>0$. In addition, we have $w_{t}=\left(u_{t}, v_{t}\right) \in$ $C^{0}\left(\left[0, T_{0}\right] ; H_{r}^{2}(\mathbb{R})\right) \times C^{0}\left(\left[0, T_{0}\right] ; H_{r}^{2}(\mathbb{R})\right)$.

\section{DISCUSSIONS}

The results are interesting when the stability and their corresponding interactions are analyzed in complementary form for the examples indicated in reference [1].

\section{CONCLUSIONS}

Throughout this work, it has been used an infinitesimal generator of a strongly continuos semigroup(see Pazy,A.[7]). Whereas the local existence of the solutions of a coupled system of BenjaminBona-Mahony (BBM) type in a weighted Sobolev space with initial data, it was considered the domain of 
the infinitesimal generator as a Banach space equipped with a suitable norm and so, we have defined a mapping $\Phi$ which it was proved within the main result, as being a contraction mapping the closed $R$-ball $\left(Y_{R}(T)\right)$ in $X(T)=C^{0}\left([0, T] ; H_{r}^{2}(\mathbb{R})\right) \times C^{0}\left([0, T] ; H_{r}^{2}(\mathbb{R})\right)$ into itself. Hence, we found a unique fixed point $w(t)$ for the operator $\Phi$ within the ball of radius $R$. On the other hand, it also was proved that $w$ is in fact differentiable and solve the initial value problema

$$
\left\{\begin{array}{l}
A w_{t}+B w_{x}+(F w)_{x}=0 \\
w(x, 0)=w_{0}(x)
\end{array}\right.
$$




\section{REFERENCES}

[1] Chen, H and X. Wang. Stability of the solitary wave solutions to a coupled BB system, J. Diff. Eq., 2016

[2] Albert,J. and Bona,J. "Comparisons between model equations for long waves" Journal nonlinear Sci 1(1991) pp. 345-374.

[3] Bisognin,V. "On the asymptotic behavior of the solutions of a nonlinear dispersive system of BenjaminBona -Mahonytype” Bolletino UMI 7 10-B(1996), pp. 99-128.

[4] Bona,J.-Ponce,G.-Saut,J.C. "A model system for strong interaction between internal solitary waves" Comm. Math. Phys. Appl.Math.,143(1992) pp. 287-313

[5] Bisognin,,E-Bisognin,V-Perla,G., "Asymptotic behavior in time of the solutions of a coupled system of Kdv equations”, Funkcialaj Ekvacioj, 40(1997) pp 353-370.

[6] Renardy,M. and Rogers,R.. “An introduction to partial differential equations”Springer Verlag N.Y. 1993

[7] Pazy,A., "Semigroup of linear operators and applications to partial differential equations", Springer Verlag N.Y. 1983

[8] Lions,J.L. “Quelques methods de resolution des problemes aux limites non lineaires ”,Dunod Gauthier Villars, Paris, 1969

[9] Miller,J.R., and Weinstein, M.I. "Asymptotic stability of solitary waves for the regularized Long. Waves equation”. Comm.Pure Appl. Math. 49(1996) 399-441

[10]Medeiros, L.A., and Milla,.M. "Weak solutions for a nonlinear dispersive equation". J.Math. Anal. Appl. 59(1977).,432-441

[11] J P. Albert, j.l. Bona , D. B. Henry Sufficent conditions for stability of solitary -wave solutions of model equations for long waves . Phys. D, 1987.

[12] Peregrine,D. H. " Calculations of the development of an undular bore" Journal Fluid Mechanic 25, parte 2 (1966)321-330

[13] Tsutsumi,M. "Weighted Sobolev space and rapidly decreasing solutions of some non linear dispersive wave equations" J. Diff. , 42, 1981. 